\title{
Pulsar radio spectra and the interstellar scintillation
}

\author{
Valerij M. Malofeev \\ Pushchino Radio Astronomy Observatory of P.N.Lebedev Physical \\ Institute, 142292, Pushchino (Moskow reg), Russia
}

\begin{abstract}
A review of the pulsar radio spectra is continued. Two approches of the spectrum construction are considered. The influence of the interstellar stcintillations on the pulsar flux density measurements and spectra is examined. The description of new pulsar spectra catalog is made. Examples of different types of spectra including one for Geminga are presented.
\end{abstract}

\section{Introduction}

The analysis of a spectrum is one of the main tools to understand the emission mechanism of any cosmic source. Attempts to obtain pulsar spectra have been made from the begining of pulsar investigations. The review of the main publications is given by Malofeev (1996). These observations showed that pulsar spectra are well described by power laws of the form $S \propto \nu^{-\alpha}$ with $\alpha$ between 0.6 and 2.9. Moreover, the first observations showed a significantly different value of $\alpha$ and flux density variations up to $100 \%$ at time scales from seconds to weeks (e.g. Cole et al. 1970) There are basically two possible explainations for these fluctuations: intrinsic variation of the pulsar radio emission and the evident influence of the interstellar scintillation on the pulsar signal (see for example Sieber 1973).

To distinguish these two alternatives several simultaneous observations have been made at a number frequencies (see references in Malofeev 1996). Correlated variations of flux density over a wide frequency range would be expected in the case of an intrinsic mechanism, which was not observed for the main part of pulsars. However, these observations gave a number of very interesting results. These are breaks in the spectra of PSR B0950+08 and B1133+16 and lowfrequency turnovers in the spectra of about ten objects. The general power law form of the spectra was, however, confirmed.

Most of the flux density variations may be caused by interstellar scintillation, but a small part of the data shows correlated changes of flux density while keeping the shape of the spectra in the range from 16 to $1400 \mathrm{MHz}$, as it shown in Fig. 1 for PSR B0329+54 (Kuzmin et al. 1978). Simultaneous observations showed, that the averaged spectra corresponded to different form presented in the publication of Sieber (1973, a dashed line in Fig.1). This conclusion has two consequences. The first one is, that the spectrum variations, as a rule, are random and averaging of the flux density measurements at the given observing frequency in a suitable way gives a stable form of the pulsar spectrum. The sec- 


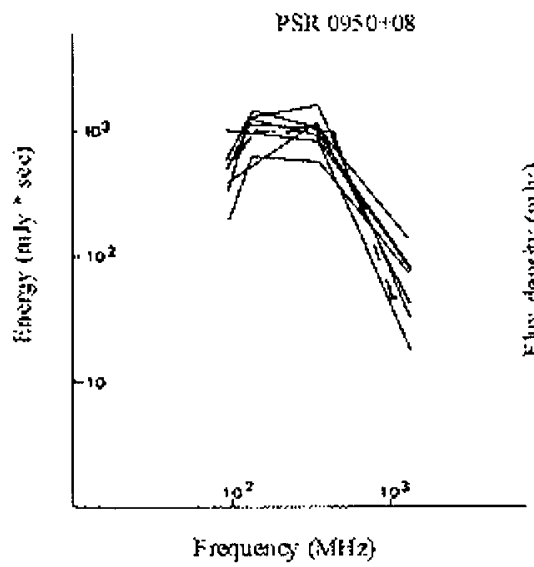

Fig I Sinultaneous spect ra in seven of the days, the dhenvations were made dusing $G$ minl.

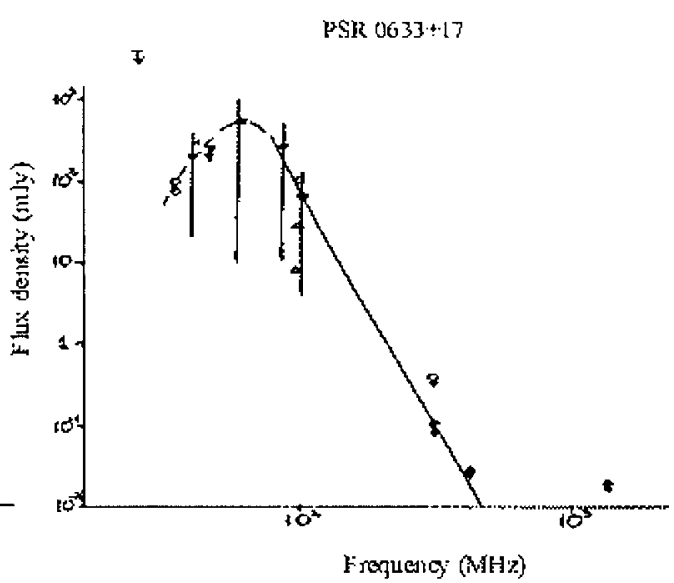

Fiy 2 Comtgiled Geminga sjectrum Our drevations: at 50.60 .87 and $102 \mathrm{MH}$ : $\mathrm{K}$ anacliandran ot al 1998 : at 75 and $318 \mathrm{MH} z$ : Xijou ris (mivate comnn.) at a $7 \mathrm{WH}$ Burder ot al. 1999: at 317,410 and $1500 \mathrm{NH}$; Ul'sando (jwivate omust.) at $25 \mathrm{MHH}$.

ond consequence is that it is possible to use not only simultaneous observations. One may reduce the effects of refractive and diffractive interstellar scintillation by averaging flux densities over a long time interval or using many measurements of different authors made at different epochs.

\section{Two approaches}

There are two approaches to the pulsar spectra investigation. The first one is a study of the original pulsar spectra based on own observations, using a few frequencies over a narrow range, for examle: PSR B1919+21 at $0.09-2.7 \mathrm{GHz}$ (Robinson et al., 1968); 10 PSR's at 0.25-8.1 GHz (Backer \& Fisher, 1974); 343 PSR at $0.4-1.6 \mathrm{GHz}$ (Lorimer et al. 1995). In this case the authors must be careful with a conclusion about the form of pulsar spectra. They must be sure that their measurements reflect real values of the pulsar flux density, but not the casual values, caused by interstellar scintillation. This type of research gives possibility to estimate the spectral index in some frequency range, but their statistical analysis requires using data for the whole radio frequency range, taking into account a low-frequency turn-over and a high-frequency break in spectra.

The second approach consists of the construction of the compiled spectra in a possible wider frequency range. Such spectra were presented for the first time by Sieber (1973) and this approch was continued by Malofeev \& Malov 


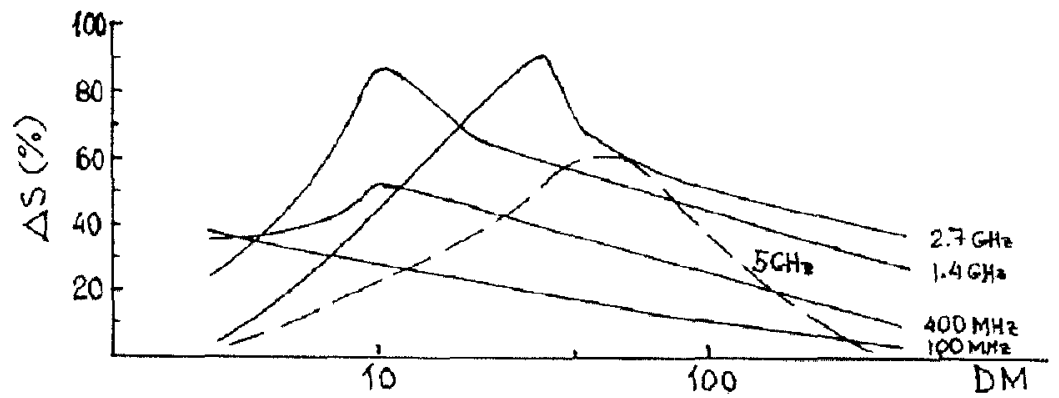

Fig. 3 The estimation of the flux density error coused by the scintillation effects
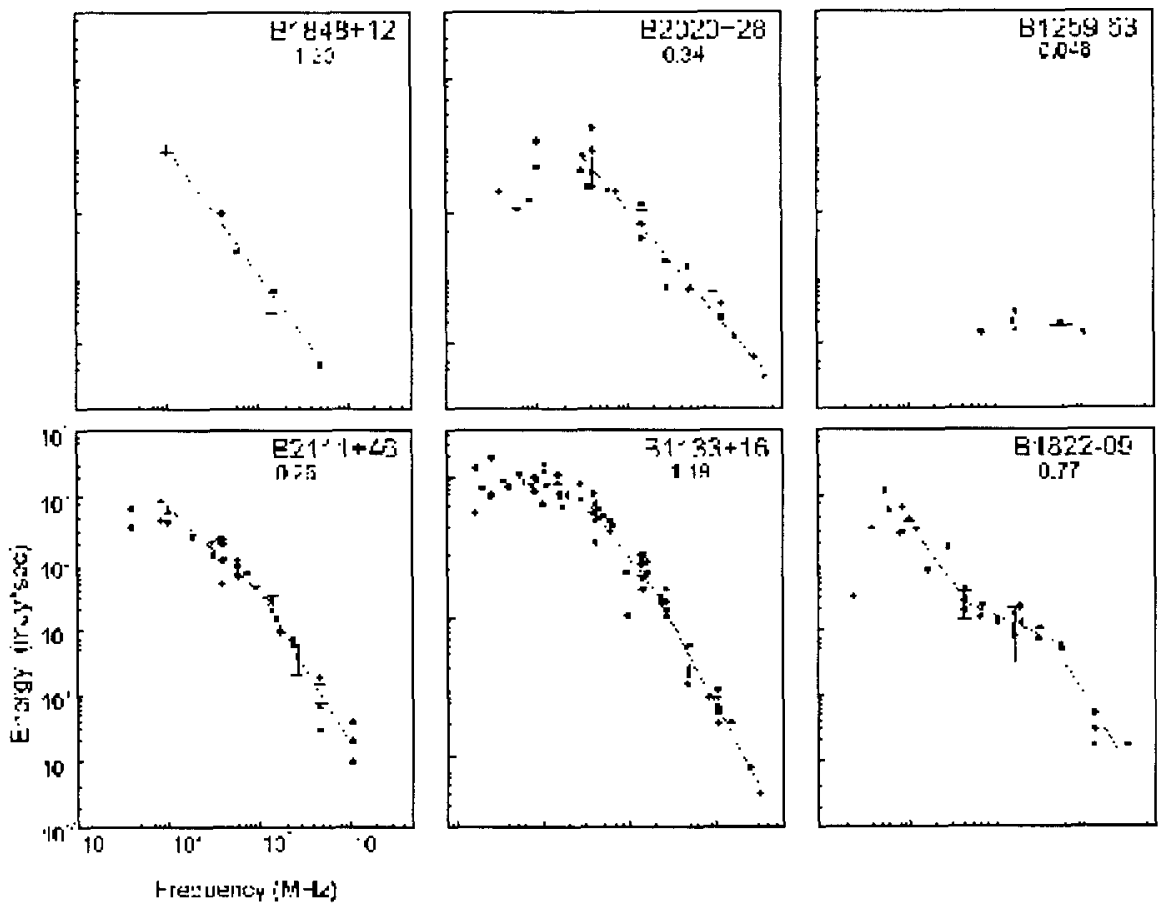

Fig.4 The eramples of the different types of pultar spectre 
(1980) and Malofeev et al. (1994). These papers contain a few tens of objects. Our last catalog presents 336 compiled spectra, including 151 ones which were constructed in a wider frecuency range (Malofeev 1999).

\section{The estimation of scintillation effects}

To obtain compiled spectra taking into account the errors of the energy measurements we meet two problems. The first one is the absence of any error estimation for the large part of the published data, and second one is that of given energy uncertainties do not take scintillation effects into account, although particularly this effect gives the main contribution to the uncertainties (for example, Lyne $\&$ Rickett 1968). To estimate the scintillation influence, one must know a few scintillation parameters at every observing frequency. Some of them have been measured for few dozen of a strong pulsars only (e.g., Cordes 1986).

Pulsar observations allow to measure the next scintillation parameters: the scintillation index $(m)$, the decorrelation time $(t)$ and the decorrelation bandwidth $(\Delta \nu)$. There are two ways to estimate the scintillation effects for many pulsars over a wide frequency range. One way is the use of a theoretical prediction, second ones is the use the empirical dependencies, obtained on the base of the measured scintillation parameters for diffractive and refractive scintillation. The theoretical predictions have a strong dependence on the unknown form of the three-dimensional spectrum of the electron density fluctuations in the interstellar medium (see for example Rickett 1990)

We used a second way. In the work by Shishov et al. (1995) the statistical dependencies of the scintillation parameters versus the frequency and a dispersion measure $(D M)$ have been obtained on the base of many published measurements, as a rule, in the strong scintillation regime, for example Cordes (1986) and the references in the paper Shishov et al. (1995). We included all available data, obtained in the weak regime too (for examle Wolszczan et al. 1974 and Malofeev et al. 1996). The estimation of the critical frequency between the strong and the weak regimes was made using a statistical dependence: $\nu_{c}(G H z)=D M \cdot 0,1$ (Pynzar' \& Shishov 1980). All statistical dependencies for diffractive $\left(m_{d}, \Delta \nu_{d}, t_{d}\right)$ and refractive $\left(m_{r}, t_{r}\right)$ scintillations are presented by Malofeev et al. (2000). These empirical curves reflect the complex character of the decorrelation bandwidth $\Delta \nu_{d}$ and the decorrelation time $t_{d}$ (defined as half of the $\mathrm{ACF}$ at half the maximum value) dependencies.

The relative error of the flux density caused by the scintillation effects was estimated as (Malofeev et al. 2000):

$$
\Delta S=\left[\left(\Delta S_{t_{r}} \cdot m_{r}\right)^{2}+\left(\Delta S_{t_{d}} \cdot m_{d} \cdot m(M)\right)^{2}\right)^{1 / 2}
$$

Here $\left.m^{2}=\left(<(S-\bar{S})^{2}\right\rangle / \bar{S}^{2}\right)$ - the scintillation index and

$$
\Delta S_{t_{r}} \text { or } \Delta S_{t_{d}} \simeq 1 /\left(t / 3 t_{m}\right)^{1 / 2}
$$

where $t$ - is the period of observations and $t_{m}=t_{d}$ or $t_{r}$. The error of the inadequate smoothing of the scintillation effects in the wide frequency band $\left(\Delta \nu_{R}\right)$ has been obtained by Sutton (1971): 


$$
m(M)=m / m_{0}=\left((2 / M) \arctan M-\left(1 / M^{2}\right) \ln \left(1+M^{2}\right)\right)^{1 / 2} .
$$

Here $M=\Delta \nu_{R} / \Delta \nu_{d}$, and $m$ and $m_{0}$ - are the scintilation indices, when a signal was received by the rectangle form of the frequency band and the infinite narrov ones.

Using values $m_{r}, m_{d}, t_{d}, \Delta \nu_{d}$ (Malofeev et al. 2000) and the formulae (1-3) the estimation of $\Delta S$ has obtained for a number of the observing frequencies and for five values of $D M$ (Fig.3). The standart values of $t$ and $\Delta \nu_{R}$ has been accepted: the bandwidth $0.5,20,100,200$ and $300 \mathrm{MHz}$ has been used at 0.1 , $0.4,1.4,2.7$, and $5 \mathrm{GHz}$ accordingly. Time of the observations has been taken as $30 \mathrm{~min}$. at $100 \mathrm{MHz}$ and as $60 \mathrm{~min}$. at the all other frequencies. The dependence $\Delta S(D M)$ has a complex character at the high frequencies, where the transition from the strong to the weak scintillation regime is located. These curves give the possibility to make the estimation of $\Delta S$ for every pulsar.

\section{Catalog of pulsar radio spectra}

Our new catalogue (Malofeev 1999) was made on the basis of our own energy measurements which were combined with many other published data at all radio frequencies from $10 \mathrm{MHz}$ until $87 \mathrm{GHz}$ at the time interval between 1968 and 1998. More than 130 papers with a flux density measurement have been used for this purpose. Our catalog containts pulsars with available data at three or more well separated frequencies $\left(\nu_{h} / \nu_{l}>3\right)$, where $\nu_{h}$ and $\nu_{l}$ are the higher and the lower frequency. The examples of the different types of spectra are presented at Fig. 4. The expected scintillation effects are given by the error bars, as a rule, at the frequencies $100(400)$ and $1400(2700) \mathrm{MHz}$.

A analisys of spectra has been made at the base of the power-law dependence $E(\nu)$, as was shown in the previous works (Sieber 1973 and Malofeev et al. 1994). The low-frequency turn-over has been detected in the spectra of 102 pulsars. All spectral indexes, the break frequencies and frequencies of maximum $\left(\nu_{b}, \nu_{m}\right)$ are presented in this catalog. We include in a classification only 151 pulsar with the rich spectra obtained over a wide frequency range $\left(\nu_{h} / \nu_{b} \geq 40\right)$. The spectra of $67 \%$ of pulsars can be modelled by a simple power law - class S (PSR B1848+12 and $\mathrm{B} 2020+28$ ), while $32 \%$ of all pulsars apparently require two power laws class B (PSR B2111+48 and B1133+16 in Fig. 4). The third class of spectra $\mathrm{C}$ (complex) has only PSR B1822-09 at present time, see Fig.4. This type of spectra can be presented by three power laws. The subclass $\mathrm{S}^{m}, \mathrm{~B}^{m}$ and $\mathrm{C}^{m}$ show the spectra with low-frequency turnover (for example PSR B2020+28, $\mathrm{B} 1133+16$ and $\mathrm{B} 1822-09)$ and new subclass $\mathrm{B}_{t}^{m}$ has been established to mark spectra with a turn-up at high-frequency (Wielebinski et al. 1993 and Morris et al. 1997), for example PSR B0355+54. The most flat and most steep spectrum demonstrate PSR B1259-63 (Fig.4) and PSR B0633+17 - Geminga (Fig.2). This pulsar has low-frequency turn-over $(<70 \mathrm{MHz})$ and very narrow spectrum. The error bars show the mean root square of the flux density values. The estimation of the spectral index gives $\alpha>=5$ using our measurements and a high limits of the flux dencity obtained at 318,327 and $410 \mathrm{MHz}$ by Burderi et al. (1999) and Ramachandran et al. (1998). 
The main study of pulsar spectra parameters $\left(\alpha, \alpha_{1}, \alpha_{2}, \nu_{m}\right.$, and a number of correlations $\alpha(P), \alpha_{1}(P), \nu_{m}(P), \alpha(T), \alpha_{1}(T), \alpha(L)$, and $\alpha_{1}(L)$ has been made in first paper (Malofeev 1996). Here $T$ - characteristic age and $L$ - the integrated luminosity (Malov et al. 1994). We plan to continue statistical analisys of the spectra parameters and the search of other dependencies to study the spectrum form evolution with the time.

Acknowledgments. Author acknowledge support of the Russian Grant RFBR No 97-02-17372, INTAS Grant No 96-0154 and DFG Travel Grant.

\section{References}

Backer D.C., Fisher F. 1974, ApJ, 189, 137.

Burderi L., Fanci F., Boriakoff V. 1999, ApJ, 512, L59

Cole T.W., Hess H.K., Page C.G. 1970, Nature, 225, 712.

Cordes J.M. 1986, ApJ, 311, 183.

Kuzmin A.D., Malofeev V.M., Shitov Yu.P., Davies J.G., Lyne A.G., Robson B. 1978, MNRAS, 185, 441.

Lorimer D.R., Yates J.A., Lyne A.G., Gould D.M. 1995, MNRAS, 273, 411.

Lyne A., Rickett B. 1968, Nature, 218, 326.

Malofeev V.M., Malov O.I., Shchegoleva N.V. 2000 AZh(in press).

Malofeev V.M., Malov I.F. 1980, AZh, 57, 90.

Malofeev V.M., Gil J.A., Jessner A., Malov I.F., Seiradakis J.H., Sieber W., Wielebinski R. 1994, A\&A, 285, 201.

Malofeev V.M. 1996, in IAU Colloq. 160, Pulsars: Problems and Progress ed. Johnston S., Walker M.A., Bailes M. (Sun Francisco: PASP), 271.

Malofeev V.M., Shishov V.I., Sieber W., Jessner A., Kramer M., Wielebinski R. 1996, A\&A, 308, 180.

Malofeev V.M. Catalog of pulsar radio spectra, Pushchino, Russia, Pushchino Research Center Press, 1999; A\&A, 2000 (in press)

Malov I.F., Malofeev V.M., Sen'e D.S. 1994, AZh, 71, 762.

Morris D., Kramer M., Thum C. et al. 1997, A\&A, 322, L17.

Pynzar' A.V., Shishov V.I. 1980, AZh, 57, 1187.

Ramachandran R., Deshpande A.A., Indrani C. 1998, A\&A, 339, 787.

Robinson B.J., Cooper B.F.C., Gordes F.F., Wielebinski R. 1968, Nature, 218, 1143 .

Rickett, B.J. 1990, ARA\&A, 28, 561.

Shishov V.I., Malofeev V.M., Pynzar' A.V., Smirnova T.V. 1995, AZh, 72, 485.

Sieber W. 1973, A\&A, 28, 237.

Sutton J.M. 1971, MNRAS, 151, 51.

Wielebinski R., Jessner A., Kramer M. et al. 1993, A\&A, 272, L13.

Wolszczan A., Hesse K.U, Sieber W. 1974, A\&A, 37, 285. 